\title{
Increased Expression of Fibroblast Growth Factors in a Rabbit Skeletal Muscle Model of Exercise Conditioning
}

Nathan G. Morrow, William E. Kraus, James W. Moore, R. Sanders Williams, and Judith L. Swain

Departments of Medicine, Microbiology, and Immunology, Duke University Medical Center, Durham, North Carolina 27710

\begin{abstract}
Increased tonic contractile activity from exercise or electrical stimulation induces a variety of changes in skeletal muscle, including vascular growth, myoblast proliferation, and fast to slow fiber type conversion. Little is known about the cellular control of such changes, but pleiotropic biochemical modulators such as fibroblast growth factors (FGFs) may be involved in this response and thus may be regulated in response to such stimuli. We examined the regulation of FGF expression in an in vivo model of exercise conditioning previously shown to exhibit vascular growth and fast to slow fiber conversion. FGFs were extracted by heparin-affinity chromatography from extensor digitorum longus muscles of adult rabbits subjected to chronic motor nerve stimulation at $10 \mathrm{~Hz}$. Growth factor activity (expressed in growth factor units [GFUs]) of muscle stimulated for 3 and $21 \mathrm{~d}$ was assayed by $\left[{ }^{3} \mathrm{H}\right]$ thymidine incorporation in 3T3 fibroblasts and compared with that present in the contralateral unstimulated muscle. A small increase in heparin-binding mitogenic activity was observed as early as $3 \mathrm{~d}$ of stimulation, and by 21 d mitogenic activity increased significantly when normalized to either wet weight (stimulated, $287 \pm 61 \mathrm{GFU} / \mathrm{g}$; unstimulated, $145 \pm 39 \mathrm{GFU} / \mathrm{g}$ ) or to protein (stimulated, 5.3 $\pm 1.1 \mathrm{GFU} / \mathrm{mg}$; unstimulated, $2.2 \pm 0.6 \mathrm{GFU} /$ mg) ( $\pm \mathrm{SE}, P<0.05)$. Western analysis demonstrated increased amounts of peptides with immunological identity to acidic and basic FGFs in stimulated muscle. The increase in FGF content observed in this study is synchronous with neovascularization, myoblast proliferation, and fast to slow fiber type conversion previously shown in this model. These results demonstrate that increased expression of FGFs is associated with motor nerve stimulation and increased tonic contractile activity of skeletal muscle, and suggests that these proteins may play a regulatory role in the cellular changes that occur during exercise conditioning. ( $J$. Clin. Invest. 1990. 85:1816-
\end{abstract} 1820.) fibroblast growth factor $\bullet$ skeletal muscle $\bullet$ exercise

\section{Introduction}

Fibroblast growth factors (FGFs) ${ }^{1}$ have been demonstrated to act as regulatory molecules in a number of cellular processes.

Address correspondence to Dr. Judith L. Swain, Duke University Medical Center, Box 3828, Durham, NC 27710.

Received for publication 14 September 1989 and in revised form 20 December 1989.

1. Abbreviations used in this paper: $\mathrm{EDL}$, extensor digitorium longus; FGF, fibroblast growth factor; GFU, growth factor unit.

J. Clin. Invest.

(c) The American Society for Clinical Investigation, Inc. $0021-9738 / 90 / 06 / 1816 / 05 \$ 2.00$

Volume 85, June 1990, 1816-1820
Both acidic and basic FGFs have been purified to homogeneity and their biological functions extensively characterized (reviewed in references $1-3$ ). The cDNAs encoding these heparin-binding growth factors have been cloned and found to be members of a multigene family that includes several protooncogenes. Results from in vitro studies suggest that FGFs may be involved in a variety of processes, including the control of angiogenesis, the regeneration of injured tissues, the maintenance of the differentiated or undifferentiated state of cells, and the induction of mesodermal structures in the developing embryo.

Though FGFs have been postulated to be endogenous mediators of vascular growth, muscle regeneration, and gene expression, their actual role in vivo is unclear. Little is known about the mechanisms by which they exert their effects, or about the regulation of expression of these proteins. To examine the potential importance of FGFs to these processes in vivo, we used a rabbit skeletal muscle model of exercise conditioning in which angiogenesis, fast to slow fiber conversion, and satellite cell proliferation are known to occur (4-6). We looked for temporally associated changes in FGF expression during skeletal muscle conditioning, as muscle FGF content might be expected to change if FGFs are involved in mediating any or all of these events.

Previous studies have documented the presence of heparin-binding mitogens in skeletal muscle $(7,8)$. Our results demonstrate that these growth factors increase in abundance in muscle during motor nerve stimulation. This increased mitogenic activity is synchronous with vascular growth and is similarly associated with changes in fiber characteristics. As assessed by Western analysis, the increase in heparin-binding mitogenic activity primarily reflects increased quantities of acidic FGF, but basic FGF is also increased as assessed by Western analysis. Since striated myocytes are known to be rich in acidic FGF (9) while other cell types found in muscle are not (1-3), these data suggest that skeletal muscle cells are the primary source of the observed increase in acidic FGF. We hypothesize that the local production of FGF with exercise conditioning may regulate, through both autocrine and paracrine mechanisms, such processes as neovascular growth, cellular proliferation, and selective changes in gene expression within muscle fibers.

\section{Methods}

In vivo electrical stimulation of skeletal muscle. Miniature electrical pulse generators were inserted into the subcutaneous tissue of the hind flank of adult New Zealand White rabbits and electrodes were tunneled to the common peroneal nerve of one hind limb. Details of this procedure were as published $(10,11)$. Continuous electrical stimulation at $10 \mathrm{~Hz}$ produced palpable contractions of the anterior compartment muscles in the operated limb without otherwise affecting behavioral or locomotor activity. Stimulation at $10 \mathrm{~Hz}$ was similar to that occurring naturally in innervated slow-twitch postural muscles and 
recruited all motor units, producing changes qualitatively similar to, but more extreme than, those resulting from standard exercise conditioning (11). Stimulation of only one limb allowed matched comparisons within the same animal and controlled for humoral influences and biological heterogeneity between animals. Stimulation was maintained for $3 \mathrm{~d}(n=5)$ or $21 \mathrm{~d}(n=6)$, at which time vascular growth and fiber conversion was initiated and/or ongoing (4, 5, 12). Animals were killed and the extensor digitorum longus (EDL) muscles were dissected, weighed, rinsed free of blood with sterile saline, frozen in liquid $\mathbf{N}_{2}$, and stored at $-70^{\circ} \mathrm{C}$ until the time of extraction. Cross-sectional samples were chosen at random from the central portion of the muscle to ensure equal representation of fibrous and muscular components in all samples.

Extraction of heparin-binding mitogens from tissue samples and determination of growth factor activity. The affinity of FGFs for heparin permits the partial purification of these growth factors by heparinaffinity chromatography (13). The amount of functional protein thus purified can be assessed by measuring mitogenic activity on fibroblasts. 200-500-mg samples of EDL muscle were weighed, pulverized in liquid $\mathrm{N}_{2}$, and homogenized in $4 \mathrm{ml}$ of $10 \mathrm{mM}$ Tris, $100 \mathrm{mM} \mathrm{NaCl}$ at $\mathrm{pH}$ 7.4. Heparin-binding mitogens were released into the soluble fraction by the addition of $0.1 \%$ trypsin to the homogenate, followed by incubation at $37^{\circ} \mathrm{C}$ for $1 \mathrm{~h}$. The suspension was then centrifuged at $8,000 \mathrm{~g}$ at $4^{\circ} \mathrm{C}$ for $30 \mathrm{~min}$, and the pellet was reserved for quantitation of DNA content. An aliquot of the supernate was set aside for later determination of protein content, and the remainder was subjected to heparinaffinity chromatography as previously described (13). Samples were applied to $1 \mathrm{ml}$ heparin-Sepharose columns (Pharmacia, Uppsala, Sweden) and allowed to equilibrate for $16 \mathrm{~h}$ at $4^{\circ} \mathrm{C}$ under gentle agitation. Columns were washed with 10 column volumes of $0.6 \mathrm{M} \mathrm{NaCl}$ $10 \mathrm{mM}$ Tris, followed by elution of heparin-binding mitogens with 2.5 $\mathrm{ml}$ of $2 \mathrm{M} \mathrm{NaCl}, 10 \mathrm{mM}$ Tris at pH 7.4.

Aliquots of elutant were used immediately for determination of mitogenic activity on quiescent Balb/c 3T3 fibroblasts as described elsewhere (13). Fibroblasts were plated at 15,000 cells per well onto 96-well plates in DME containing $10 \%$ calf serum. Cells were grown to confluence and the medium was depleted of growth factors over 7-10 d. The medium was changed to DME without serum supplement on the day of the assay and $1 \mu \mathrm{Ci}$ of $\left[{ }^{3} \mathrm{H}\right]$ thymidine (sp act $2.0 \mathrm{Ci} / \mathrm{mmol}$; NEN Research Products, Wilmington, DE) was added to each well. 5 $\mu$ l of test sample or negative control $(2 \mathrm{M} \mathrm{NaCl})$ was added to each of eight wells, as was $10 \mu \mathrm{l}$ of calf serum as a positive control. Incorporation of $\left[{ }^{3} \mathrm{H}\right]$ thymidine was determined after $48 \mathrm{~h}$. Mitogenic activity is expressed in growth factor units (GFUs), with 1 GFU defined as the amount of test substance required to stimulate one-half the maximal $\left[{ }^{3} \mathrm{H}\right]$ thymidine incorporation of calf serum. Mitogenic activity was normalized to four different parameters: soluble protein, wet weight, DNA, and total muscle weight.

Quantitation of protein and DNA. The protein content of the supernate applied to the heparin-Sepharose column was determined by the colorimetric BCA method (Pierce Chemical Co., Rockford, IL). The pellet from the extraction procedure was solubilized in $1 \mathrm{ml}$ of 0.5 $\mathrm{M} \mathrm{NaOH}$ at $90^{\circ} \mathrm{C}$ for $1 \mathrm{~h}$ and DNA content was quantified colorimetrically by the diphenylamine reaction of Burton (14).
Western blot analysis. Extracts from pooled muscle samples of equivalent wet weight and protein content from EDL muscle stimulated for $21 \mathrm{~d}$, and contralateral unstimulated muscle were pooled and partially purified by heparin-affinity chromatography as described above. The elutants were concentrated sixfold by size exclusion in a Centricon 10 microconcentrator tube (Amicon Corp., Danvers, MA) by centrifugation at $5,000 \mathrm{~g}$ for $2 \mathrm{~h}$. Samples were electrophoresed on a $15 \%$ polyacrylamide gel and transferred to nitrocellulose, and Western analysis was performed. The primary antibodies used to detect FGFlike proteins were a rabbit polyclonal antibody raised against human acidic FGF (a gift from Michael Jaye and Robert Friesel, Rorer Corp., King of Prussia, PA) and a rabbit polyclonal antibody raised against human recombinant basic FGF (Synergen, Boulder, $\mathrm{CO}$ ). Immunoreactive bands were detected using a biotinylated second antibody and the alkaline phosphatase reaction (Vector Laboratories, Inc., Burlingame, CA). Recombinant acidic (human) and basic (bovine) FGF were electrophoresed concurrently as positive controls.

Statistical analysis. Comparisons of FGF content per protein, wet weight, DNA, and total muscle weight between stimulated and contralateral limbs were performed using the $t$ test for paired values. The data were expressed as mean $\pm \mathrm{SE}$.

\section{Results}

Heparin-binding mitogenic activity is increased in electrically stimulated rabbit skeletal muscle. FGF content of stimulated and control muscle samples was normalized to wet weight, soluble protelin content, and DNA content. Because changes occur in protein and DNA content during stimulation (Table I) $(10,15)$, total muscle FGF content was also calculated by multiplying GFUs per gram wet weight times the whole muscle weight.

Mitogenic activity showed a slight, but not statistically significant, increase in three of four animals after $3 \mathrm{~d}$ of stimulation when normalized to protein content (Fig. $1 a$ ), and in four of five animals when normalized to wet weight (Fig. $1 b$ ). Decreasing activity relative to DNA content at $3 \mathrm{~d}$ (Fig. $1 c$ ) is explained by an increase in mean DNA content per gram wet weight at this time point (Table I) $(P<0.005)$. This increase in DNA content has been observed previously (15) and may reflect endothelial and satellite cell proliferation, changes in connective tissue content, and possibly inflammation (6).

By $21 \mathrm{~d}$ the mean content of heparin-binding mitogens in stimulated versus unstimulated limbs had increased relative to all normalization parameters with the differences in mean FGF content per milligram protein $(P<0.02)$ (Fig. $1 a)$ and per gram wet weight $(P<0.01)$ (Fig. $1 b)$ reaching statistical significance. Mitogenic activity per milligram protein and per gram wet weight increased in each animal examined. Mitogenic activity per gram DNA increased in five of six animals, yet the differences failed to reach statistical significance $(P$

Table I. Effects of Stimulation on Weight, Protein Content, and DNA Content of EDL Muscle

\begin{tabular}{|c|c|c|c|c|c|}
\hline & \multicolumn{5}{|c|}{ Duration of stimulus } \\
\hline & \multicolumn{3}{|c|}{$3 d$} & \multicolumn{2}{|c|}{$21 \mathrm{~d}$} \\
\hline & Unstimulated & . & Stimulated & Unstimulated & Stimulated \\
\hline Muscle weight $(g)$ & $1.7 \pm 0.1$ & & $1.8 \pm 0.2$ & $2.3 \pm 0.2$ & $1.6 \pm 0.1^{*}$ \\
\hline Protein/wet weight $(\mathrm{mg} / \mathrm{g})$ & $74 \pm 9$ & & $73 \pm 8$ & $83 \pm 21$ & $65 \pm 13$ \\
\hline DNA/wet weight $(\mathrm{mg} / \mathrm{g})$ & $0.30 \pm 0.03$ & & $0.49 \pm 0.06^{\ddagger}$ & $0.48 \pm 0.11$ & $0.72 \pm 0.13$ \\
\hline
\end{tabular}

Values are expressed as the mean \pm SE. $* P<0.05,{ }^{\ddagger} P<0.005$ (stimulated versus unstimulated). 


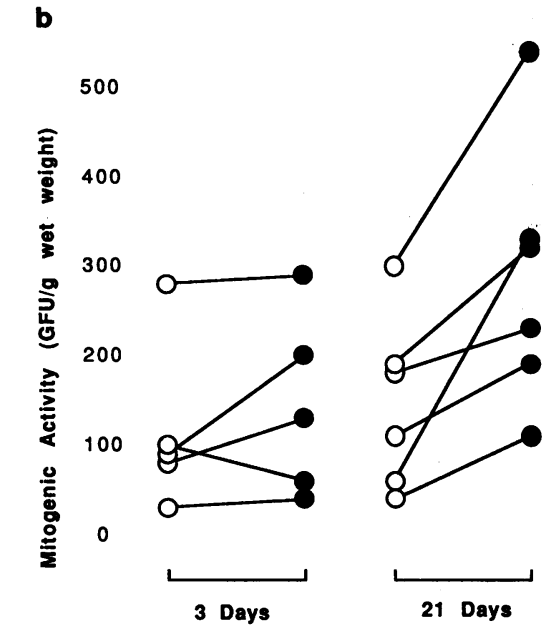

c

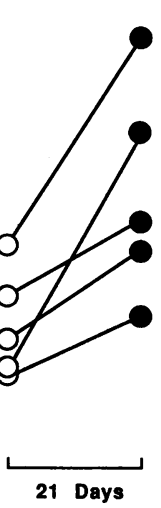

d
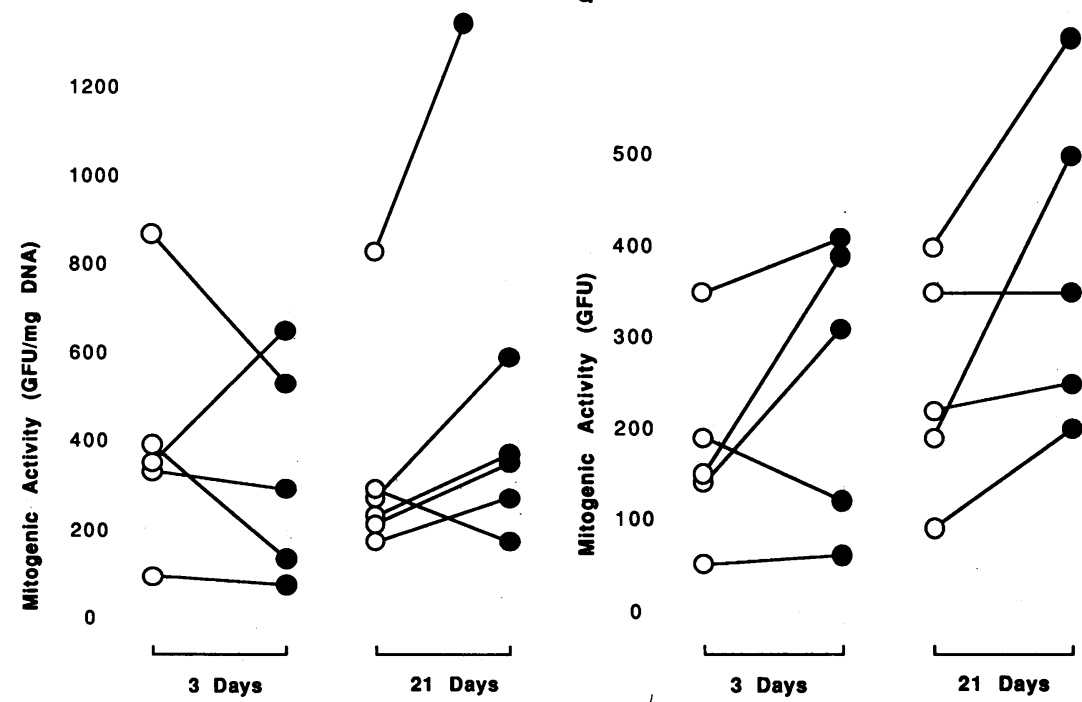

Figure 1. Effects of 3 and $21 \mathrm{~d}$ of electrical stimulation $(10 \mathrm{~Hz})$ of contractile activity on heparin-binding mitogenic activity in rabbit EDL muscle. Heparin-binding mitogenic activity, expressed as GFUs, is normalized to soluble protein content $(a)$, wet weight $(b)$, and DNA content $(c)$ of the muscle sample. Total muscle GFU content of the EDL muscle is shown in $d$. Open circles represent heparin-binding mitogenic activity from the unstimulated EDL muscle of individual rabbits and are connected by a line to closed circles representing values obtained from the stimulated limb of the same animal. Values on the ordinate are expressed in GFU per milligram protein, per gram wet weight, per milligram DNA, and per total EDL muscle.

$<0.12)$ due to inordinately high DNA levels in the stimulated limb of one animal (Fig. 1 c). When the content of heparinbinding mitogens contained in the whole EDL muscle of each limb was calculated (Fig. $1 d$ ), mean activity in the stimulated limbs increased in four of five animals $(P<0.08)$ despite a significant decrease in mean whole muscle weight of the stimulated limb $(P<0.03)$ (Table I). These data demonstrate an increase in expression of heparin-binding mitogens in vivo during $21 \mathrm{~d}$ of continuous electrical stimulation.

The heparin-binding mitogens in skeletal muscle share immunological identity with acidic and basic FGF and increase with stimulation. At least two forms of FGF (acidic and basic) elute from heparin-affinity columns at $\mathrm{NaCl}$ concentrations between 0.6 and $2 \mathrm{M}(1-3)$. Both forms are found in skeletal muscle $(7,8)$. To examine the immunological identity of the heparin-binding mitogens extracted from rabbit EDL muscle, Western analysis using antibodies to acidic and basic FGF was performed on pooled, partially purified samples from companion limbs of two rabbits stimulated for $21 \mathrm{~d}$. The results are shown in Fig. 2. A 14-kD peptide recognized by an antibody to acidic FGF (Fig. $2 a$ ) is present in unstimulated muscle and is increased 5-10-fold in stimulated muscle. Immunodetection with an antibody directed against basic FGF (Fig. $2 b$ ) demon-

strates the presence of a $24-\mathrm{kD}$ peptide in both limbs, with slightly greater amounts in the stimulated compared with control muscle. Other less abundant peptides were observed that also increased in abundance with stimulation. These results demonstrate that heparin-binding mitogens present in rabbit skeletal muscle include proteins similar in size and immunological reactivity to both acidic and basic FGF, and these proteins increase in abundance with electrical stimulation of muscle.

\section{Discussion}

The skeletal muscle model used in these experiments displays many of the processes in vivo that FGFs are known to promote in vitro, including angiogenesis, myoblast proliferation, and selective changes in gene expression within myocytes (4-6, 9, $10,15)$. We hypothesized that if FGFs mediate these events in vivo, then increased expression of FGFs should be synchronous with the observed cellular changes. The results of this study demonstrate that FGFs are regulated in vivo and that increased expression of FGFs temporally corresponds to these biological changes. While the temporal association of increased quantities of FGF with the cellular and physiologic 

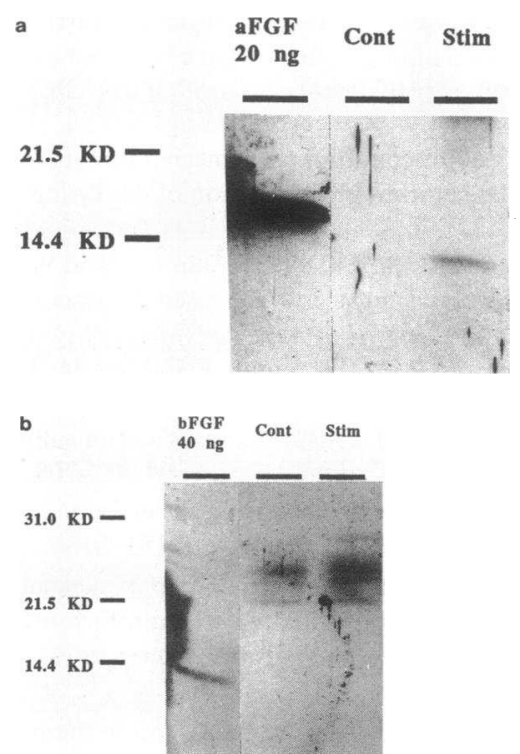

Figure 2. Western analysis of partially purified heparin-binding mitogens from the unstimulated EDL muscle of two animals (Cont) and from the contralateral EDL muscle of the same animals stimulated for $21 \mathrm{~d}$ (Stim). Starting sample weights and protein contents were equivalent for control and stimulated samples. Purified samples were concentrated sixfold by size exclusion in a Centricon $10 \mathrm{mi}$ croconcentrator tube centrifuged at $5,000 \mathrm{~g}$ for $2 \mathrm{~h}$. Samples were electrophoresed on $15 \%$ polyacrylamide gels, transferred to nitrocellulose, and incubated with polyclonal antisera to acidic $(a)$ or basic $(b)$ FGF. The molecular weight standards are shown on the left of each panel. Purified recombinant human acidic and bovine basic FGF were used as positive controls.

changes that occur in this model does not prove a causative role for FGF, it does provide corroborative evidence that FGFs may mediate some of the effects of chronic exercise conditioning.

Many changes that occur in rabbit fast-twitch EDL muscle with chronic nerve stimulation at $10 \mathrm{~Hz}$ mimic those that occur with endurance training $(11,12)$. Hudlika et al. examined changes in fiber profile and capillary density during muscle stimulation applied for $8 \mathrm{~h} / \mathrm{d}$. They showed a significant increase in capillary density beginning at $4 \mathrm{~d}$ of stimulation and continuing through $28 \mathrm{~d}$ of stimulation $(4,5)$. As FGFs are known to stimulate each of the discrete steps by which angiogenesis occurs, including endothelial cell proliferation, migration, and protease production, the increased FGF expression demonstrated in this study may indicate a primary role for this angiogenic agent in the vascular growth occurring with electrical stimulation of skeletal muscle. Although capillary growth is well documented in the rabbit model, in other species the effects of chronic electrical stimulation on muscle blood flow may differ from results observed in the rabbit (16).

Other changes observed in this model potentially are related to increased FGF expression as well. Satellite cell proliferation has been observed to occur in muscle during electrical stimulation and mechanical loading $(6,17)$. Since FGFs are known mitogens for satellite cells in vitro (18), they may function in vivo (8) to induce satellite cell proliferation. Differential expression of mitochondrial genes and nuclear genes encoding mitochondrial proteins may contribute to mitochondrial biogenesis and fiber conversion that occur in this model $(10,15,19)$. FGFs have been shown to differentially regulate expression of sarcomeric $\alpha$-actin isoforms in myoblasts in vitro (20), and therefore may serve a similar regulatory function in fiber-type conversion.

The question arises as to which cell type or types (i.e., myocyte, endothelial cell, or fibroblast) synthesize FGFs in muscle in response to stimulation. Our results indicate that some, if not most, of the increase in FGF can be attributed to the acidic form. This increase in acidic FGF probably is contributed by the muscle cells since striated myocytes are known to synthesize acidic FGF (9), while endothelial cells and fibroblasts produce little if any acidic FGF (1-3). It is unclear, however, whether satellite cells or fully differentiated myotubes are responsible for the demonstrated increase in acidic FGF. We have found that progression of differentiation correlates with decreasing quantities of acidic FGF mRNA in L6 skeletal myocytes (unpublished observations). Thus proliferating satellite cells may be the primary source of increased FGF observed in stimulated muscle.

A possible mechanism by which FGF content is increased with stimulation is as follows. FGF present in muscle is initially liberated from storage sites in the matrix (8) by mechanical forces such as stretch, or by soluble factors. Acting via specific cell surface receptors, FGFs thus liberated might induce vascular growth and satellite cell proliferation. The proliferation of satellite cells capable of synthesizing FGF could in turn result in the increased quantities of FGF observed after 21 $\mathrm{d}$ of stimulation. As such, stimuli leading to continued release of FGF from sites of sequestration in the extracellular matrix might perpetuate this cycle until biochemical homeostasis is achieved.

In summary, we demonstrated that increased expression of FGFs is temporally associated with vascular growth, satellite cell proliferation, and fast to slow fiber type conversion in an in vivo rabbit model of exercise conditioning. We suggest that FGFs may thus be involved in mediating some of these effects. Further studies to elucidate the mechanisms regulating these complex processes will have important implications for a number of pathological states involving skeletal muscle and other organ systems. The selective inhibition of vascular growth, for example, could prevent neovascular retinopathies or tumor growth. Conversely, selective stimulation of FGF expression might enhance wound healing or the regeneration of injured or diseased skeletal muscle (21), or accelerate the development of collateral channels to ischemic tissues in atherosclerotic and diabetic vascular disease. Such therapeutic strategies are contingent upon a more complete understanding of the intracellular mechanisms governing these biological processes.

\section{Acknowledgments}

This work was supported in part by National Institutes of Health grants HL-26831 to Dr. Swain and HL-35639 to Dr. Williams, and by a grant from the John A. Hartford Foundation Geriatrics Scholars Program to Dr. Morrow. J. L. Swain and R. S. Williams are Established Investigators of the American Heart Association, and W. Kraus is an American Heart Association Clinician Scientist.

\section{References}

1. Gospodarowicz, D., N. Ferrara, L. Schweigerer, and G. Neufeld. 1987. Structural characterization and biological functions of fibroblast growth factor. Endocr. Rev. 8:95-114.

2. Folkman, J., and M. Klagsbrun. 1987. Angiogenic factors. Science (Wash. DC). 235:442-447.

3. Burgess, W. H., and T. Maciag. 1989. The heparin-binding (fibroblast) growth factor family of proteins. Annu. Rev. Biochem. 58:575-606. 
4. Hudlika, O. 1982. Growth of capillaries in skeletal and cardiac muscle. Circ. Res. 50:451-461.

5. Hudlika, O., L. Dodd, E. M. Renkin, and S. D. Gray. 1982. Early changes in fiber profile and capillary density in long-term stimulated muscles. Am. J. Physiol. 243:H528-H535.

6. Maier, A., B. Gambke, and D. Pette. 1986. Degeneration-regeneration as a mechanism contributing to the fast to slow conversion of chronically stimulated fast-twitch rabbit muscle. Cell Tissue Res. 244:635-643.

7. Kardami, E., D. Spector, and R. C. Strohman. 1985. Myogenic growth factor present in skeletal muscle is purified by heparin-affinity chromatography. Proc. Natl. Acad. Sci. USA. 82:8044-8047.

8. Yamada, S., N. Buffinger, J. Dimario, and R. C. Strohman. 1989. Fibroblast growth factor is stored in fiber extracellular matrix and plays a role in regulating muscle hypertrophy. Med. Sci. Sports Exercise. 21:S173-S180.

9. Weiner, H. L., and J. L. Swain. 1989. Acidic fibroblast growth factor mRNA is expressed by cardiac myocytes in culture and the protein is localized to the extracellular matrix. Proc. Natl. Acad. Sci. USA. 86:2683-2687.

10. Williams, R. S., S. Salmons, E. A. Newsholme, R. E. Kaufman, and J. Mellor. 1986. Regulation of nuclear and mitochondrial gene expression by contractile activity in skeletal muscle. J. Biol. Chem. 261:376-380.

11. Salmons, S., and G. Vrbova. 1969. The influence of activity on some contractile characteristics of mammalian fast and slow muscles. J. Physiol. (Lond.). 201:535-549.

12. Salmons, S., and J. Henriksson. 1981. The adaptive response of skeletal muscle to increased use. Muscle \& Nerve. 4:94-105.
13. Shing, X., J. Folkman, R. Sullivan, C. Butterfield, J. Murray, and $M$. Klagsbrun. 1984. Heparin affinity: purification of a tumor-derived capillary endothelial cell growth factor. Science (Wash. DC). 223:1296-1298.

14. Burton, K. 1956. Study of the conditions and mechanism of the diphenylamine reaction for the colorimetric estimation of deoxyribonucleic acid. Biochemistry. 62:315-323.

15. Williams, R. S., M. Garcia-Moll, J. Mellor, S. Salmons, and W. Harlan. 1987. Adaptation of skeletal muscle to increased contractile activity. J. Biol. Chem. 262:2764-2767.

16. Acker, M., W. A. Anderson, R. L. Hammond, F. DiMeo, Jr., J. McCullum, M. Staum, M. Velchik, W. E. Brown, D. Gale, S. Salmons, and L. W. Stephenson. 1987. Oxygen consumption of chronically stimulated skeletal muscle. J. Thorac. Cardiovasc. Surg. 94:702-709.

17. Schultz, E. 1989. Satellite cell behavior during skeletal muscle growth and regeneration. Med. Sci. Sports Exercise. 21:S181-S186.

18. Allen, R. E., and K. A. Boxhorn. 1989. Regulation of skeletal muscle satellite cell proliferation and differentiation by transforming growth factor- $\beta$, insulin-like growth factor- 1 , and fibroblast growth factor. J. Cell. Physiol. 138:311-315.

19. Williams, R. S. 1986. Mitochondrial gene expression in mammalian striated muscle. J. Biol. Chem. 261:12390-12394.

20. Parker, T. G., S. Packer, and M. Schneider. 1989. Peptide growth factors can provoke "fetal" contractile gene expression in cultured cardiac myocytes. Clin. Res. 37:284A. (Abstr.)

21. DiMario, J., N. Buffinger, S. Yamada, and R. C. Strohman. 1989. Fibroblast growth factor in the extracellular matrix of dystrophic (mdx) mouse muscle. Science (Wash. DC). 244:688-690. 